\section{The in-house psychologist: do we speak the same language? Short report of a qualitative practice project}

\author{
Birgitte Schoenmakers, ${ }^{1,2}$ \\ Jan De Lepeleire ${ }^{2}$ \\ 1Department of Public Health and \\ Primary Care, University Leuven and \\ ${ }^{2}$ Academic Teaching Practice, Leuven, \\ Belguim
}

\section{Abstract}

Interdisciplinary collaboration is gaining importance. Although general practices (GP's) have a comprehensive experience in collaboration with psychologists, research on this topic is scarce. In house referrals to a psychologist are assumed to lower the thresholds for patients and GP's. In this study it was investigated whether the GP's reasons to refer in were accor dance with the treatment strategy of the residing psychologist. The study is performed in a retrospective, observational cross section design. The studied population were the residing psychologist and GP's. Both were asked to complete a questionnaire. Outcome measures where the referral reasons of the GP's and the treatment strategy of the psychologist. A total sample of 92 patients of 6 GP's was studied. Over $60 \%$ of the patients were referred for counseling but only in $25 \%$ of the cases this proposal was carried out by the psychologist. Overall, the referral reasons of the GP's were not in accor dance with the treatment strategy of the psychologist. A close collaboration and communication between general practitioners and psychologists is both difficult and indispensable. This practice research demonstrated that the referral motives of the GP's usually do not correspond to the treatment policy of the psychologist. This observation is partly explained by a lack of understanding of the GP in the treatment strategies of the psychologists. Another part of the explanation is that there is a pre-selection of the GPs referrals rather influenced by patient characteristics than by pathology.

\section{Introduction}

There has been some progress in multidisciplinary collaboration in general practice in recent years. Considerable experience has already been acquired in several European countries on various collaboration frameworks with psychologists. ${ }^{1}$ There is, however, a lack of well-designed studies to support and justify the development of these initiatives. Although it is assumed that the number of referrals to specialist services will be reduced as a result, there is no research offering consistent confir mation of this. ${ }^{2}$ On the one hand there is evidence that an in-house psychologist has little influence on the number of referrals to specialist services. ${ }^{1}$ There is little difference between the reasons for referral to a psychologist working within a general practice (GP) and an outside psychologist. 3 On the other hand the threshold for referrals is lowered when there is an in-house psychologist. ${ }^{2}$ GPs also indicate that despite the rise in the number of consultations for psychosocial problems, the accessibility of consultations with a psychologist is the critical threshold. 4,5 In contrast to earlier observations on the increased prevalence of psychosocial consultations, the number of psychological diagnoses has fallen and the number of referrals has risen during the past 10 years. ${ }^{2} \mathrm{GPs}$ are clearly quite reticent when they encounter psychosocial problems and seek support from specialist services. ${ }^{6,7}$ In this context, opportunities for discussion with psychologists are highly valued and the quality of collaboration is usually judged to be high when the psychologist works within the practice.

From the perspective of a patient requesting psychosocial help, it is interesting to observe that a majority of these patients consult their GP first and then receive a focused referral to a psychologist. ${ }^{8}$ On the other hand, only onethird of patients within the population as a whole would discuss psychosocial problems with their GP. ${ }^{9}$ It should not be surprising that the support offered by the psychologist is usually more successful if there is easy and open communication with the referring GP.10 A number of studies have also shown that the use of psychotropic medication is reduced dur ing the intervention by the psychologist, although this effect disappears when the consultations come to an end (Robson 1984, Pharoah 1995, Sibbald 1996, data not shown).

Following on from earlier research, this study aims to outline the reasons or objectives for referral by GPs of patients with psychosocial problems in the context of a collaboration framework with an in-house psychologist.

\section{Materials and Methods}

\section{Definition}

The in-house psychologist in the academic GP teaching practice is defined as a care provider based within that GP practice. He can be consulted without a referral. The collaboration framework involves a minimal number of conditions, which are defined in discussions between the psychologist and the group of GPs in accordance with the existing literature. By
Correspondence: Birgitte Schoenmakers, University Leuven, Department of Public Health and Primary Care, Academic Teaching Practice, Kapucijnenvoer 33, block J, box 7001, Leuven, Belgium.

E-mail: birgitte.schoenmakers@med.kuleuven.be

Key words: primary health care, mental health services, general practice.

Acknowledgements: the authors would thank Wouter Van Mechelen, Marleen Brems, Sabine Van Baelen, Geert Van Boxem and Kristel Vandeput.

Conflict of interest: the authors declare potential conflict of interests.

Received for publication: 17 October 2012.

Accepted for publication: 16 December 2012

This work is licensed under a Creative Commons Attribution NonCommercial 3.0 License (CC BYNC 3.0).

(C) Copyright B. Schoenmakers and J. De Lepeleire, 2013

Licensee PAGEPress, Italy

Health Psychology Research 2013; 1:e9 doi:10.4082/hpr.2013.e9

consensus the psychologist is available within the practice for about 20 hours a week. This time is divided between consultations, discussions with doctors and attendance at staff meetings. The support provided is focused on symptom-oriented interventions in the case of personal and/or relational problems and major life events. The treatment provided is seen as shortterm, lasting for approximately 10 consultations. Frank or apparently serious psychiatric pathology as evaluated by the GP is excluded from the remit of the support provided. Therefore, patients with a personality disorder or acute psychosis according to the primary axis in the DSM-IV classification are excluded.

The reason for referral is divided into four categories, in accordance with the treatment strategies of the psychologist: advice and/or diagnosis, counseling, psychotherapy or support in the context of a severe psychiatric illness.

Finally, the psychologist uses by consensus two main categories to define the nature of the patients' problem (called clinical disorder): common problems (depression, major life events, adjustment disorders and problems in relationships with a partner) and less common presentations (addiction, sexually related problems, identity problems, parent-child problems, etc.).

\section{Study design and research question}

The study was set up as an observational, ret- 
rospective cross-section. The primary research question addresses the reason or objective given by the GP for the referral. In accordance with the treatment strategies adopted by the psychologist, these are subdivided into requests for advice and/or diagnosis, for counseling, for psychotherapy or for support in the context of a severe psychiatric illness. The secondary research question is whether the reason for referral corresponds to the treatment strategy followed by the psychologist.

\section{Outcome measures}

\section{and measurement tools}

The primary outcome measure is the reason for referral by the GP. The secondary outcome measure is the psychologist's treatment strategy. The psychologist listed all patients consulting in a time span of one year. This list was presented to the GP's requesting to code the reason for referral. The psychologist coded the treatment strategy for each patient. Both parties were blinded to each other's responses.

\section{Planned analysis}

The dependent variable is the reason for referral. The independent variables are the referring doctors and the nature of the problem (called clinical disorder). The clinical disorder is subdivided into common problems (depression, major life events, adjustment disorders and problems in relationships with a partner) and less common presentations. A bivariate model with Fisher Exact test was used to detect the relationship between the reason for referral and the treatment strategy of the psychologist. A logistic regression model using the maximum likelihood estimates was used to check the influence of the independent variables on the correlation between GP's reason for referral and psychologists' treatment strategy.

\section{Results}

\section{Data collection}

In this academic GP teaching practice the seven doctors make similar numbers of referrals to the psychologist. The data from one of the doctors could not be used for analysis. The other doctors retrospectively recorded their reasons for referring patients to the psychologist for support from March 2007 to April 2008. The starting date for recording the data was the date when the psychologist started work at the practice. In total the psychologist treated 92 different patients during this period.

For each of these patients the psychologist recorded the clinical complaint, the total number of visits, completion of the course and any new presentations. Most presentations were related to major life events $(n=28)$, depressive episodes $(n=10)$, relationship problems with the partner $(\mathrm{n}=10)$ and adjustment disorders $(n=9)$. In $40 \%(n=39)$ of presentations there was a personality disorder. During the course of treatment, one in four $(n=24)$ patients was referred by the GP to specialist services for further diagnostic or therapeutic advice. Approximately 1/3 $(n=33)$ of the patients stopped treatment by agreement with the psychologist and 14\% ( $\mathrm{n}=13)$ stopped abruptly.

\section{Reasons for referral and treatment strategy}

More than half $(60 \%)$ of referrals were requests for counseling. Requests for further diagnosis were made in $1 / 6$ of cases. A further
$1 / 6$ of the patients were referred for psychotherapy (Tables 1-4). Referrals were made in relation to psychiatric disorders in a minority of only four patients. For common clinical complaints, doctors refer more frequently for counseling (71\%), while for less common presentations the referral is usually for counseling, diagnosis and psychotherapeutic support (42\%, 22\%, 25\% respectively, Fisher exact $\mathrm{P}<0.05)$. The psychologist treats patients with common complaints mainly by offering psychotherapeutic support followed by counseling (respectively $42 \%$ and $33 \%$, Fisher exact $\mathrm{P}<0.005)$. In presentations with less common clinical conditions, the psychologist carries out further diagnostic work and provides psychotherapeutic support (respectively $45 \%$ and

Table 1. Frequency table reasons for referral.

\begin{tabular}{lcccc} 
Referral reason (GP) & Counseling & Diagnosis & Psychotherapy & Psychiatric disorder \\
GP1 & 11 & 1 & 4 & 1 \\
GP2 & 7 & 0 & 3 & 0 \\
\hline GP3 & 11 & 8 & 3 & 0 \\
GP4 & 11 & 2 & 1 & 0 \\
\hline GP5 & 13 & 4 & 3 & 0 \\
GP6 & 3 & 0 & 2 & 3 \\
\hline
\end{tabular}

GP, general practice.

Table 2. Frequency table for reasons for referral and treatment strategy of the psychologist.

\begin{tabular}{lcccc} 
Treatment & Counseling & Diagnosis & Psychotherapy & Psychiatric disorder \\
referral reason & & & 21 & 0 \\
Counseling & 17 & 18 & 5 & 1 \\
Diagnosis & 4 & 5 & 9 & 1 \\
\hline Psychotherapy & 1 & 5 & 2 & 1 \\
Psychiatric disorder & 0 & 1 &
\end{tabular}

Fisher exact Pr $\leq \mathrm{P} 0.0024$

Table 3. Frequency table of treatment strategy of the psychologist by clinical disorder.

\begin{tabular}{lcccc} 
Clinical disorder & Counseling & Diagnosis & Psychotherapy & Psychiatric disorder \\
Common problems & 19 & 14 & 24 & 0 \\
Others & 3 & 16 & 13 & 3 \\
\hline
\end{tabular}

Fisher exact $\operatorname{Pr} \leq \mathrm{P}$ 0,0024. Common clinical disorders (representing more than $10 \%$ of the cases): depressive episode (10\%), relational problems $10 \%$ ), life stage problems (30\%), adjustment disorder (10\%). Others (representing each less than $10 \%$ of the cases): all others

Table 4. Frequency table of clinical disorder versus reasons for referral of GP.

\begin{tabular}{lcccc} 
Clinical disorder & Counseling & Diagnosis & Psychotherapy & Psychiatric disorder \\
Common problems & 41 & 7 & 7 & 1 \\
Others & 15 & 8 & 9 & 3 \\
\hline
\end{tabular}

Fisher exact Pr $\leq \mathrm{P} 0.027$. 
$37 \%$, Fisher exact $\mathrm{P}<0.005)$.

The doctor's reason for referral is not precisely followed by the psychologist providing treatment (Fisher exact $P>0.1$ ). Just over 1/3 of the patients referred receive psychotherapeutic support and further diagnostic work is done for $1 / 3$ of patients. Counseling is only offered by the psychologist in $1 / 4$ of cases.

A logistic regression analysis (Table 5) with the GP's reason for referral as dependent variable and the treatment strategy and clinical disorder being the independent variables confirms that the relationship between referral and treatment objectives is rather poor. No significant interactions were found. Only for counseling and diagnose some weak relationship was found with the referral objective of the GP $\left(\chi^{2}=0.03\right)$. This relationship was not influenced by the clinical disorder of the patient.

\section{Discussion}

This practice study shows that the reasons for referral by the GP doctor are barely in accordance with the treatment strategy performed by the psychologist. Only in case of a GP's request for counseling or diagnose there was a poor accordance with the actual treatment proposal of the psychologist.

GPs refer for counseling in $2 / 3$ of cases, as compared with the $1 / 4$ of patients for whom counseling is actually provided.

GPs report that they are very much in need of support in relation to psychosocial problems in everyday practice. More patients are presenting with these problems, while waiting lists for mental health services are unacceptably long. ${ }^{4}$ Although GP in-service training has been offering more modules on psychotherapy for a number of years now, only a limited number of GPs deliver this treatment themselves. Part of the explanation for this is no doubt the time pressure resulting from these types of consultations. ${ }^{6}$ The remainder of the explanation could be found in the fact that GPs are less familiar with psychotherapy and more uncertain about it. ${ }^{11} \mathrm{GPs}$ still tend to offer pharmacological treatment and reserve psychotherapy for unclear or restricted clinical presentations. ${ }^{12}$

There is also little correspondence between the referral mechanisms adopted by different doctors. The reasons for referral tend to depend more on the patient's characteristics than on the actual problem. ${ }^{5,13} \mathrm{GPs}$ are quicker to refer older patients, those with a history of psychological distress and patients with significant physical illness for psychotherapy. Although this was not explicitly addressed in the research, it is assumed that the most important reason for referral by doctors is a request for counseling.2,14 In this practice study, doctors give counseling as the major rea- son for referral. There are a number of factors that may account for the fact that counseling is then offered to only $1 / 4$ of these patients. Doctors refer for counseling if the problem is more clearly defined and if the patient meets specific criteria. In other words a degree of pre-selection has already taken place, which is probably based on inadequate information from the patient or on the fact that the doctor has limited insight into psychopathology.

More than one-third of patients with psychological problems indicate that they do not want to talk to their GP about them.9,15 Younger people in particular have difficulty with the stigma and taboos surrounding psychological problems and see the familiar context of their own GP as threatening. ${ }^{12}$ Patients who want to be referred have a tendency to let their GP know this, so that they will have a better guarantee of an appropriate referral. ${ }^{8}$ Finally, GPs are not always able to pick up or decode the patient's signals, which places significant stress on the doctor-patient relationship. ${ }^{7}$

The approaches to psychosocial problems adopted by GPs and their referral patterns are generally different and in some cases very different from those of the psychologists. ${ }^{5}$ GPs usually make a referral if they have the feeling that the case goes beyond their expertise or if patients state that they want more help. The counseling that GPs then request is consequently more of a request for support for their own management, although the patient's own needs would tend to require a different approach. In this practice project it was found that psychologists delivered equal amounts of psychotherapeutic support, counseling and diagnostic work.

Harmonizing the management of support and follow-up offered to patients with psychosocial problems requires in-depth discussions between the psychologist and the GP.16 The approach to patients taken by GPs and psychologists must complement each other in order to cover all the different aspects of psychological distress. In the absence of any dis- cussion it emerges that there are clear discrepancies between these two groups of care providers in terms of both diagnosis and management.

The approach adopted by psychologists for patients with less common clinical conditions was also clearly different from the reasons for referral (diagnosis) offered by the doctor. They made diagnoses and offered psychotherapy in equal numbers of cases. Clearly the experience of the psychologist has a significant influence here. The doctor evidently feels less familiar with clinical presentations that occur less commonly and consequently tends to refer for a diagnosis more frequently. This supports the above statement that GPs mainly refer when they find themselves at the limits of their own expertise. An improved understanding of psychopathology would probably offer benefits in terms of patient referrals.

The referral pattern is strongly influenced by the relationships and arrangements that exist between the GP and the psychologist.3,17 GPs who work closely or even in-house with psychologists refer to psychiatry no less frequently than GPs without such close contacts. ${ }^{1}$ It has been found that where there is good collaboration between psychologists and GPs the number of referrals to the psychologist is significantly higher than where no agreement or collaboration framework exists. ${ }^{10}$ In this practice study it was found that very few referrals are made for psychiatric conditions. These are patients who have a known psychiatric diagnosis and were being followed up by the GP. The reason why the number is so low is probably because a considerable part of patients with a history of severe psychiatric illness are already receiving extensive in-house or outside support.

This study has a number of significant limitations. First of all the study was carried out retrospectively. The GPs and the psychologist were asked to complete questionnaires on a retrospective basis. Their responses may therefore be colored by their subsequent contacts with the patients and discussions with the psychologist.

Table 5. Logistic regression analysis for relationship and interaction between reason for referral, treatment strategy and clinical disorder.

\begin{tabular}{lccc} 
& \multicolumn{2}{c}{ Maximum likelihood estimates } & Pstimates \\
Strategy counseling & -1.2 & 4.4 & 0.03 \\
Strategy diagnosis & -1.3 & 4.4 & 0.03 \\
\hline Strategy psychotherapy & -0.6 & 1.2 & 0.3 \\
Common clinical disorder & -0.16 & 0.2 & 0.6 \\
\hline Interaction counseling & -0.9 & 1.7 & 0.2 \\
Interaction diagnosis & -0.7 & 1.8 & 0.2 \\
\hline Interaction psychotherapy & 0 & - & -
\end{tabular}

Dependent variable: reason for referral; independent variables and interaction terms: clinical disorder, treatment strategy; convergence criterion satisfied. 
On the other hand, both the doctors and the psychologist keep very meticulous patient records and none of the data gathered was subjective. Secondly there is no control group. Ideally the same study should have been carried out in a similar practice but without a resident psychologist in order to draw robust conclusions about referral patterns. As a result of this there is a significant selection bias at the practice level. The team of six GPs, however, offers sufficient heterogeneity to keep the risk of selection bias as low as possible.

Thirdly, there was no questioning of the GPs and the psychologist to produce a qualitative description of barriers to collaboration and benefits of collaboration. Thresholds to collaboration are well-known and are often the result of lack of communication and time constraints.

\section{Conclusions}

Close collaboration between GPs and psychologists is often seen by those involved as both absolutely necessary and simultaneously difficult. The position of psychologists within primary health care is important in order to guarantee that patients are referred appropriately. Since GPs and patients almost unavoidably face long waiting lists for psychosocial support, an in-house psychologist seems to offer a solution in practice. This practice study has shown that the reasons for referral by the doctor usually do not match the objective for the psychologist's own treatment management. This observation is explained to some extent by the lack of insight among GPs into the psychologist's approach. The explanation is also partly linked to the fact that GPs undertake some pre-selection of their referrals. It is not necessarily the patients with the greatest need for help who find their way to the psychologist.

Further research should be carried out to demonstrate the mechanisms underlying referrals, thereby yielding a pattern for effective referrals.

\section{References}

1. Ashworth M, Clement S, Sandhu J, et al. Psychiatric referral rates and the influence of on-site mental health workers in general practice. Br J Gen Pract 2002;52:39-41.

2. Cape J, Parham A. Relationship between practice counselling and referral to outpatient psychiatry and clinical psychology. Br J Gen Pract 1998;48:1477-80.

3. Tata P, Eagle A, Green J. Does providing more accessible primary care psychology services lower the clinical threshold for referrals? Br J Gen Pract 1996;46:469-72.

4. Clatney L, Macdonald H, Shah SM. Mental health care in the primary care setting: family physicians' perspectives. Can Fam Physician 2008;54:884-9.

5. Sigel P, Leiper R. GP views of their management and referral of psychological problems: a qualitative study. Psychol Psychother 2004;77:279-95.

6. Raine R, Lewis L, Sensky T, et al. Patient determinants of mental health interventions in primary care. Br J Gen Pract 2000;50:620-5.

7. Gulbrandsen P, Hjortdahl P, Fugelli P. General practitioners' knowledge of their patients' psychosocial problems: multipractice questionnaire survey. BMJ 1997;314:1014-8.

8. Kovess-Masfety V, Saragoussi D, SevillaDedieu C, et al. What makes people decide who to turn to when faced with a mental health problem? Results from a French survey. BMC Public Health 2007;7:188.

9. Bushnell J, McLeod D, Dowell A, et al. Do patients want to disclose psychological problems to GPs? Fam Pract 2005;22:631-7.

10. Hull SA, Jones C, Tissier JM, et al. Relationship style between GPs and community mental health teams affects referral rates. Br J Gen Pract 2002;52:101-7.

11. Schulte TJ, Isley E, Link N, et al. General practice, primary care, and health service psychology: concepts, competencies, and the combined-integrated model. J Clin Psychol 2004;60:1011-25.

12. Bushnell J, McLeod D, Dowell A, et al. The treatment of common mental health problems in general practice. Fam Pract 2006;23:53-9.

13. Raine R, Lewis L, Sensky T, et al. Patient determinants of mental health interventions in primary care. Br J Gen Pract 2000;50:620-5.

14. Jorm AF, Korten AE, Jacomb PA, et al. Beliefs about the helpfulness of interventions for mental disorders: a comparison of general practitioners, psychiatrists and clinical psychologists. Aust N Z J Psychiatry 1997;31:844-51.

15. Freiberg-Golvan D, Fradkin P, McCormack $\mathrm{H}$, et al. Working together. General practitioners and psychologists. Aust Fam Physician 1988;17:998-9.

16. Salmon P, Stanley B, Milne D. Psychological problems in general practice patients: two assumptions explored. Br J Clin Psychol 1988;27:371-9.

17. Younes N, Gasquet I, Gaudebout P, et al. General practitioners' opinions on their practice in mental health and their collaboration with mental health professionals. BMC Fam Pract 2005;6:18. 